\title{
Joint Manifestations in Children with Familial Mediterranean Fever in Armenia: Clinical and Genetic Characteristics
}

\author{
Gayane Amaryan ${ }^{1, ~ *, ~ G a y a n e ~ K h l o y a n ², ~ T a m a r a ~ S a r k i s i a n ~}{ }^{3}$, Artashes Tadevosyan ${ }^{4}$, \\ Rotraud Katharina Saurenmann ${ }^{5}$ \\ ${ }^{1}$ National Pediatric Centre for Familial Mediterranean Fever of “Arabkir" Medical Complex - Institute of Child and Adolescent Health, \\ Department of Pediatrics N 2 of Yerevan State Medical University, Yerevan, Armenia \\ ${ }^{2}$ Rheumatology Service of “Arabkir” Medical Complex - Institute of Child and Adolescent Health, Yerevan, Armenia \\ ${ }^{3}$ Centre of Medical Genetics and Primary Health Care; Department of Medical Genetics of Yerevan State Medical University, Yerevan, \\ Armenia \\ ${ }^{4}$ Department of Public Health and Healthcare Organization of Yerevan State Medical University, Yerevan, Armenia \\ ${ }^{5}$ Department of Child and Adolescent Medicine, Kantonsspital Winterthur, Winterthur, Switzerland
}

Email address:

gayaneamaryan@yahoo.com (G. Amaryan), gayaneamaryan@gmail.com (G. Amaryan)

${ }^{*}$ Corresponding author

\section{To cite this article:}

Gayane Amaryan, Gayane Khloyan, Tamara Sarkisian, Artashes Tadevosyan, Rotraud Katharina Saurenmann. Joint Manifestations in Children with Familial Mediterranean Fever in Armenia: Clinical and Genetic Characteristics. International Journal of Immunology. Vol. 9, No. 2, 2021, pp. 22-28. doi: 10.11648/j.iji.20210902.11

Received: March 13, 2021; Accepted: April 6, 2021; Published: April 16, 2021

\begin{abstract}
Familial Mediterranean fever (FMF) is an autosomal-recessive autoinflammatory disease in the group of Hereditary periodic fever syndromes (HPFS), characterized by recurrent, self-limited attacks of fever and polyserositis, which last 2-4 days. It manifests mainly in childhood and often has early onset, resolve without sequels. Genetic testing of FMF is efficient and allows diagnosing of atypical cases. FMF is a significant health care problem in Armenia because of high frequency of carriers of MEFV mutations 1:3 - $4(0.21)$ and marked FMF prevalence 54.7 per $10 \times 000$ of the total population. Articular symptoms are found in about $45 \%$ of cases. They usually manifest as acute recurrent arthritis (ARA) or arthralgia, but more rarely also chronic arthritis can be found. We aimed to investigate clinical and genetic characteristics of the joint manifestations in Armenian children with FMF. The charts of all 715 patients with proven diagnosis of FMF at the National Pediatric Center (NPC) for FMF were reviewed for joint manifestations. The diagnosis of FMF and disease severity were determined according to the Tel-Hashomer criteria and molecular-genetic detection of $12 \mathrm{MEFV}$ mutations common for Armenians. The characteristics of joint manifestations and overall FMF disease were then compared to $M E F V$ mutation analysis. There were 438 boys and 277 girls with an age at diagnosis between 3 months and 17 years (mean age: $8.64 \pm 0.17$ ). Joint involvement was observed in $56.4 \%$ of all 715 cases. The manifestation was ARA in $30.5 \%$, arthralgia in $21.2 \%$ and chronic arthritis (CA) in $4.7 \%$, which would also qualify for a diagnosis of JIA. The frequency of ARA was associated with M694V mutation, mainly M694V homozygous and M694V heterozygous genotypes. The risk of CA depended on $M E F V$ genotype of the FMF patients and was the highest in M694Vheterozygotes and M694Vhomozygotes. M694V heterozygous genotype was noticed significantly more frequently among FMF patients with spondyloarthritis in comparison to those without it. The probability of the development of CA among FMF patients without M694V mutation was significantly higher also in heterozygotes in comparison to compound-heterozygotes. We concluded, that the frequency of joint involvement among Armenian pediatric FMF patients was high - 56.4\%, especially for CA. Chronic arthritis may be the first manifestation of FMF and occurred usually in patients with severe M694V mutations. Patients of Armenian origin with refractory arthritis should be asked for isolated febrile attacks, hemorrhagic vasculitis, episodes of pleuritis and family history to rule out FMF.
\end{abstract}


Keywords: Familial Mediterranean Fever, Children, Joint Manifestations, Clinical and Genetic Characteristics

\section{Introduction}

The Familial Mediterranean fever (FMF; OMIM 249100) is the most common autosomal recessively inherited monogenic autoinflammatory disease in the group of Hereditary periodic fever syndromes (HPFS) [3-5]. It is characterized by recurrent, self-limited attacks of fever and painful aseptic serosal inflammation in the peritoneum, pleura or synovia, elevated acute phase reactants. FMF symptoms generally last for 2-4 days and resolve without sequels. The most severe complication of it - is secondary amyloidosis, if not treated [4, 6]. FMF has an especially high prevalence among certain ethnic groups of Mediterranean origin such as Sephardic Jews, Armenians, Arabs, and Turks. But also in non-Mediterranean basin countries FMF is quite frequent due to active migration, increased number of mixed marriages, and other factors [7].

FMF is significant health care problem in Armenia, because of high frequency of carriers of MEFV gene mutations 1:3 - 4 (0.21) and marked FMF prevalence - 54.7 per $10 ` 000$ of the total population [8-11]. It manifests mainly in childhood, often has early or atypical onset.

The MEFV gene, responsible for FMF, is involved in inflammatory reactions through altered leukocyte apoptosis, secretion of interleukin -1beta (IL-1b), activation of the NFkappa B pathway and thereby the degree of inflammation. The concomitant presence of $M E F V$ mutations in other than FMF diseases may modify their presentation and severity [8, 25, 26]

Articular involvement is the third major FMF manifestation (after febrile peritonitis and pleuritis), and is found in about $45 \%$ of cases [6,12-15]. It usually presents as acute recurrent arthritis (ARA) of the large joints of the lower or upper extremity (hip, knee, ankle, wrist) and/or arthralgia. There are three characteristic features of the ARA of FMF: (1) a very high fever in the first 24 hours, (2) involvement of one large joint of the lower extremity, and (3) gradual resolution of the signs and symptoms after peaking in 24-48 hours, leaving no sequelae; often a sterile synovial effusion is present [16]. ARA may last longer than other clinical FMF manifestations, however, a prolonged course of arthritis lasting more than 1-2 weeks is rare.

Arthralgia is considered an FMF symptom if it occurs in the context of typical FMF attacks. Transient arthralgia is usually short lasted and more often involves large joints.

Another articular manifestation of FMF is chronic arthritis (CA) which occurs in $2-5 \%$ of FMF cases. It may be confused with other chronic arthritis of childhood such as juvenile idiopathic arthritis (JIA), because it most commonly affects the large joints of the lower extremity, but sometimes also features resembling spondyloarthritis (sacroileitis, enthesitis) can be found [6]. Investigation of these manifestations is important to better describe and learn more about the overlap between the
FMF, spondyloarthritis and JIA.

\section{Material and Methods}

\subsection{Study Setting and Data Collection Methods}

The aim of this study was to investigate clinical and genetic characteristics (phenotype-genotype correlations) of the joint manifestations in Armenian children with FMF. Between 1997 and 2008, all 715 children (438 boys and 277 girls, 1.5:1) followed at the National Pediatric Center for Familial Mediterranean Fever (FMF NPC) of "Arabkir" Medical Complex - Institute of Child and Adolescent Health by a single investigator (GA) (from a total of 1800 FMF patients) were included into this follow-up study. The charts were reviewed and the initial FMF diagnosis and severity of clinical FMF features were determined according to the TelHashomer criteria [1, 2].

\subsection{Genetic Analysis}

$M E F V$ mutation analysis was performed at the Centre of Medical Genetics of Armenia. In total, $12 \mathrm{MEFV}$ mutations and corresponding 23 genotypes were identified by reverse hybridization, restriction analysis, PCR, and sequencing [911]. For the purpose of this study the patients were grouped into 5 relatively homogeneous groups according to the presence of the highly penetrate M694V mutation, responsible for the most severe clinical features.
1) Homozygotes
(M694V/M694V;
M680I/M680I; V726A/V726A);
2) M694V heterozygotes (M694V/0);
3) M694V compound-heterozygotes (M694V/Other);
4) Other compound-heterozygotes (Other/Other);
5) Other heterozygotes (Other/0)

\subsection{Definitions of the Articular Involvements}

The following definitions of types were used for this study:

1) Articular involvement was defined as any symptom from the joints not explained by other factors (e.g. trauma, infection, mechanical joint problems)

2) Arthralgia was defined as severe pain in one or more joints occurring in the context of a typical, highly febrile FMF attack but not fulfilling the definition for arthritis used in the (International League of Associations of Rheumatology (ILAR) classification criteria for juvenile idiopathic arthritis [17].

3) Acute recurrent arthritis (ARA) was defined as an acute arthritis (fulfilling the above mentioned ILAR definition) of one or several joints occurring in the context of a typical FMF-attack and of $\leq 2$ weeks duration

4) Chronic arthritis (CA) was defined as arthritis (according to ILAR definition) of $>6$ weeks duration and with features of joint impairment such as limitation of the range of movement and/or development of 
radiologic changes. These patients would also fulfill the criteria for a diagnosis of Juvenile Idiopathic Arthritis (JIA) according to ILAR criteria.

\subsection{Statistical Analysis}

All data from patients' medical records were entered and stored in a specially developed Microsoft Access 2003 database (Microsoft Corporation, Redmond, WA). The statistical analysis was performed using Epi-Info 2000 software (CDC, Atlanta, GA). Chi-square test was used for comparison of two nominal variables in table two by two with Yates' correction for continuity.

\subsection{Ethical Considerations}

Ethical approval for the study was obtained from the Ethical Committee of the Yerevan state medical university. Each participant gave their informed consent before participating in the study, and their anonymity, privacy, and confidentiality was respected. Consent was signed and obtained after reading and understanding the content of the consent form.

\section{Results}

\subsection{Socio-demographic Characteristics of Patients}

Of the 715 patients in the database, 21 patients had no $M E F V$ test result available and in 7 patients the $M E F V$ testing had not revealed a mutation of the 12 mutations analyzed. These patients were therefore not included into the patient cohort reported in this study. Sample demographic characteristics of the 687 FMF patients reported in this study were the following: all patients were of Armenian nationality, representing all regions of the country. Two thirds of them (458 patients) lived in urban and one third (229 patients) in rural settlements. The mean age at diagnosis was 8.6 years ranging from 3 months to 17 years.

\subsection{Determining Genotypes of Patients}

The following genotypes were determined: homozygotes (237 patients) - (M694V/M694V (211); M680I/M680I (16); V726A/V726A (8); R761H/R761H (2); M694V heterozygotes: M694V/0 (61); M694Vcompound-heterozygotes: M694V/Other (257); Other compound-heterozygotes: Other/Other (93); Other heterozygotes: Other/0 (39).

\subsection{Articular Involvement}

Articular involvement was observed in 404 (56.4\%) patients, 248 boys and 156 girls. The manifestation was ARA in 218 children $(30.5 \%), \mathrm{CA}$ in 34 children (4.7\%). Arthralgia as an FMF symptom was found in 152 children $(21.2 \%)$.

In $92(12.9 \%)$ of the patients articular involvement preceeded other clinical symptoms of FMF and was the only manifestation for several years (range 1- 6 years), often associated with fever. Articular involvement presented as
ARA and rarely as CA (indistinguishable from JIA). Monoand oligoarthritis prevailed over other types of arthritis $(69 / 201)$. Predominant involvement of the knees and ankles was observed in 69/404 FMF patients (17\%). In 201 cases $(49.7 \%)$ oligoarthritis of 2 joints (symmetrical or/and asymmetrical) was diagnosed. Only in 7 (1.7\%) FMF patients polyarticular arthritis including small joints was noticed.

\subsection{Acute Recurrent Arthritis (ARA)}

ARA - mainly mono- or oligoarthritis of the large joints of the lower (knees, ankles, hips) and more rarely the upper extremity (wrists, elbows) had the typical benign clinical course known for FMF associated arthritis. It usually presented with fever and disappeared within a few days with no sequelae. In 25 patients $(6.2 \%$ of the cases) this was the first FMF manifestation, in 59 children (14.6\%) it developed later at an average of 6 years after disease onset.

We found a positive correlation of the frequency of ARA with the type of the $M E F V$ mutation: patients with homozygous mutations significantly more frequently had joint manifestations compared with other combinations (compound-heterozygous and heterozygous) (Chi square 23.41, p<0.0001). Patients with any M694V mutation (i.e. M694V/M694V; M694V/0 and M694V/others) significantly more frequently suffered from ARA, as compared to those without $M 694 \mathrm{~V}$ (other homozygotes, compound heterozygotes or other heterozygotes) (Chi square 16.51, $\mathrm{p}<0.0001)$. Also, the risk of ARA in M694V homozygote (M694V/M694V) and heterozygote (M694V/0) patients was significantly higher with Odds Ratio (OR) 2.18 (95\% CI 1.45 $\div 3.28$; Yates'corrected Chi square 15.03; $\mathrm{P}<0.0001)$ compared with compound-heterozygote variant M694V/V726A; and OR 1.94 (95\% CI $1.03 \div 3.66$; Yates' corrected Chi square 4.26; $\mathrm{P}<0.05)$ compared with a compound-heterozygote variant M694V/ M680I.

However, when we looked at individual genetic groups the highest rate of ARA (75\%) was found among V726A homozygotes (V726A/V726A). This rate was statistically significantly higher than in M694V homozygous patients $\begin{array}{llll}(\mathrm{M} 694 \mathrm{~V} / \mathrm{M} 694 \mathrm{~V}) & (41.7 \%) \quad(\mathrm{p}<0.003, & \text { Chi Square }) .\end{array}$ Interestingly, the frequency of ARA was also significantly lower in M694V homozygotes $(19.4 \%)\left(\chi^{2}=15.36, p<0.0005\right)$ compared to those with M694V compound-heterozygous constellation (M694V/V726A (22.6\%); M694V/M680I $(27.2 \%)$.

Thus, the frequency of ARA was associated with $M E F V$ genotype: V726A homozygous, M694V compoundheterozygous, M694V homozygous and M694V heterozygous genotypes might be considered a risk factor for ARA in FMF. Table 1. (The frequency of articular involvement and MEFV genotypes). Figure 1. (The risk of arthritis in children with FMF and genotypes).

\subsection{Chronic Arthritis (CA)}

Was observed in 34 FMF patients (4.7\%), who would also qualify for a diagnosis of juvenile idiopathic arthritis (JIA). 
The frequency varied from $1.1 \%$ to $13.1 \%$ depending on the genotype (Table 1). In the majority of FMF patients with CA (29/34 children; 85.3\%) genetic analyses revealed an M694V mutation. CA was especially more fréquent in M694V heterozygotes (M694V/0) with 8/61 patients (13.1\%) and M694V/M694V 9/211 patients (8.1\%) in comparison with M694V compound-heterozygotes (M694V/M680I; M694V/V726A; 10/237 children; 4.3\%). Table 2. (The

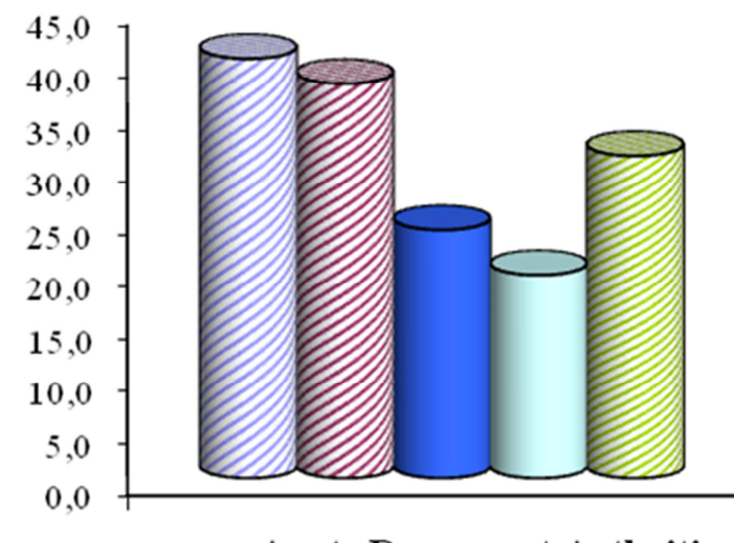

Acute Recurrent Arthritis frequency of Chronic Arthritis and MEFV genotypes in FMF patients). Thus, the risk of CA was associated with $M E F V$ genotype of the FMF patients and was higher in M694Vheterozygotes and M694V- homozygotes. The frequency of CA was significantly increased also in FMF heterozygotes without M694V mutation (10.2\%) compared to compoundheterozygotes $\left(\chi^{2=} 14.46 ; \mathrm{p}<0.006\right)$.

Figure 1. The risk of arthritis in children with FMF and genotypes.

Table 1. The frequency of articular involvement and MEFV genotypes.

\begin{tabular}{|c|c|c|c|c|c|c|c|c|}
\hline \multirow{3}{*}{$M E F V$ genotypes } & & & \multicolumn{6}{|c|}{ Type of articular involvement } \\
\hline & \multicolumn{2}{|c|}{ All patients $(n=687)$} & \multicolumn{2}{|c|}{ ARA $n=218$} & \multicolumn{2}{|c|}{ CA (JIA) $n=34$} & \multicolumn{2}{|c|}{ Arthralgia $n=152$} \\
\hline & & & Abs & $\%$ & Abs. & $\%$ & Abs & $\%$ \\
\hline Homozygotes & $237 *$ & 34.5 & 85 & 35.9 & 10 & 4.3 & 54 & 22.8 \\
\hline (M694V/M694V) & 211 & 31.0 & 77 & 36.5 & 9 & 4.2 & 42 & 19.9 \\
\hline$(V 726 A / V 726 A)$ & 8 & 1.16 & 6 & 7.0 & - & - & 2 & 25.0 \\
\hline (M680I/M680I) & 16 & 2.33 & 2 & 12.5 & 1 & 6.2 & 10 & 62.5 \\
\hline M694V/0 & 61 & 8.8 & 21 & 34.4 & 8 & 1.1 & 9 & 14.7 \\
\hline M694V/Other & 257 & 37.4 & 76 & 29.6 & 11 & 4.2 & 60 & 23.3 \\
\hline Other/Other & 93 & 13.5 & 25 & 26.8 & 1 & 1.1 & 19 & 20.4 \\
\hline Other/ 0 & 39 & & & 5.7 & 11 & 10.2 & 10 & 25.6 \\
\hline
\end{tabular}

Table 2. The frequency of Chronic Arthritis and MEFV genotypes in FMF patients.

\begin{tabular}{|c|c|c|c|c|}
\hline \multirow{2}{*}{$M E F V$ genotypes } & \multicolumn{2}{|c|}{ All patients $(n=687)$} & \multicolumn{2}{|c|}{ Chronic Arthritis (JIA) $(n=34)$} \\
\hline & Abs. & $\%$ & Abs. & $\%$ \\
\hline Homozygotes & 237 & 34.5 & 10 & 4.3 \\
\hline (M694V/M694V) & 211 & 31.0 & 9 & 8.1 \\
\hline (M680I /M680I) & 16 & 1.16 & 1 & 6.2 \\
\hline M694V/0 & 61 & 8.8 & 8 & 13.1 \\
\hline M694V/Other & 257 & 37.4 & 11 & 4.2 \\
\hline (M694V/M680I) & 88 & 12.8 & 7 & 7.9 \\
\hline$(M 694 V / V 726 A)$ & 141 & 20.52 & 4 & 2.8 \\
\hline Other/Other & 93 & 13.5 & 1 & 1.1 \\
\hline Other/ 0 & 39 & 5.7 & 4 & 10.2 \\
\hline$(V 726 A / 0)$ & 19 & 2.8 & 3 & 15.8 \\
\hline$(R 761 H / 0)$ & 2 & 0.3 & 1 & 50.0 \\
\hline
\end{tabular}

$\uparrow$ FMF patients with no $M E F V$ mutations 0/0 (7) and not investigated for mutations (21)

Joint manifestations as one of the criteria of FMF severity correlated with $M E F V$ genotypes, namely M694V homozygous (M694V/M694V) and M694V heterozygous

\section{Homozygotes \\ 曰 M694V/0 \\ 口 M694V/Other \\ $\square$ Other/Other \\ 口Other $/ 0$ \\ shaded $\mathbf{P}<0,05$}

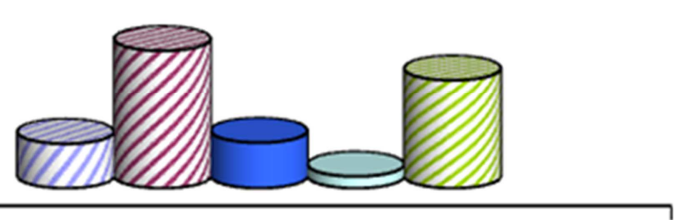

Juvenile Idiopathic Arthritis 
without M694V mutation (Other/Other) are associated with mild to moderate FMF severity. Table 3

Table 3. FMF disease severity and MEFV genotypes.

\begin{tabular}{|c|c|c|c|c|c|c|c|c|}
\hline \multirow{3}{*}{ MEFV genotypes } & & & \multicolumn{6}{|c|}{ FMF disease severity } \\
\hline & \multicolumn{2}{|c|}{ All patients $(n=687 ; 28 \dagger)$} & \multicolumn{2}{|l|}{ mild } & \multicolumn{2}{|c|}{ moderate } & \multicolumn{2}{|c|}{ severe } \\
\hline & & & Abs & $\%$ & Abs & $\%$ & Abs & $\%$ \\
\hline Homozygotes & 237 & 34.5 & 6 & 2.5 & 193 & 81.4 & 38 & 16.0 \\
\hline (M694V/M694V) & 211 & 31.0 & 2 & 0.9 & 171 & 81.0 & 38 & 18.0 \\
\hline$(V 726 A / V 726 A)$ & 8 & 1.16 & 2 & 25.0 & 6 & 75.0 & - & - \\
\hline (M680I /M680I) & 16 & 2.33 & 3 & 18.8 & 13 & 81.2 & - & - \\
\hline $\mathrm{M} 694 \mathrm{~V} / 0$ & 61 & 8.8 & 11 & 18.0 & 49 & 80.3 & 1 & 1.6 \\
\hline Others/Others & 93 & 13.5 & 21 & 22.6 & 70 & 75.3 & 2 & 2.1 \\
\hline Others/ 0 & 39 & 5.7 & 14 & 35.9 & 24 & 61.5 & 1 & 2.5 \\
\hline
\end{tabular}

Twenty two of the 34 FMF patients with CA (64.7\%) had all typical manifestations of FMF (including polyserositis), as well as a severe course of CA: polyarthritis or oligoarthritis with knee, ankle, hip joint involvement or spondyloarthritis (X-ray confirmed sacroiliitis). Six of them developed CA after 2-3 years of follow-up, mostly with signs of spondyloarthritis.

FMF patients with CA predominantly had involvement of the big joints of the lower extremity, and the majority of them (20/34) developed spondyloarthritis with symmetric sacroiliitis. HLA B27 allele was identified in 7 children. Obviously, these patients need further follow-up for differential diagnosis with ankylosing spondylitis associated with FMF.

Almost one third of the patients with JIA-like arthritis (11/34) had early manifestations of atypical (abortive) FMF presentation: recurrent febrile or afebrile arthritis/spondylitis (as the only symptom) and a positive FMF-family history. Then rare thoracic pain attacks would developed and a beneficial effect of colchicine therapy was observed. More than half of the patients with atypical FMF course and CA
(7/11) were M694V heterozygotes. Systemic onset of CA (fulfilling criteria of systemic onset JIA) occured in 2 patients, both had heterozygous genotype (M694V/0 and V726/0). Considerable growth retardation (below the $3^{\text {rd }}$ percentile) was noticed in 8 patients with CA.

Spondyloarthritis was associated with $M E F V$ genotype and ranged from $1.1 \%$ to $8.2 \%$ in the different genetic groups. The frequency was relatively higher in M694V heterozygotes in comparison with M694V homozygotes Table 4 (Genotypes of patients with spondyloarthritis - like chronic arthritis (sacroileitis). The M694V homozygous genotype was identified in 40\% FMF children with spondyloarthritis and in $30.1 \%$ of patients without it. Nevertheless, there was no statistical difference in the frequency of M694V homozygous $\left(\chi^{2}=0.49 ; \quad \mathrm{p}>0.05\right)$ and $\mathrm{M} 694 \mathrm{~V}$ compound-heterozygous genotypes (M694V/other) $\left(\chi^{2}=1.86 ; \mathrm{p}=0.17\right)$ in patients with spondyloarthritis. However, M694V heterozygous genotype (M694V/0) was found significantly more frequently among patients with spondyloarthritis compared to those without it (Fisher exact test $\mathrm{p}=0.025$ ).

Table 4. Genotypes of patients with spondyloarthritis - like chronic arthritis (sacroileitis).

\begin{tabular}{|c|c|c|c|}
\hline \multirow{2}{*}{$M E F V$ genotypes } & \multirow{2}{*}{ All patients $(n=687 ; 28 \dagger)$} & \multicolumn{2}{|c|}{ Spondyloarthritis-like chronic arthritis $(n=20)$} \\
\hline & & Abs. & $\%$ \\
\hline Homozygotes & 237 & 8 & 3.4 \\
\hline$(M 694 V / M 694 V)$ & 211 & 7 & 3.3 \\
\hline (M680I /M680I) & 16 & 1 & 6.2 \\
\hline $\mathrm{M} 694 \mathrm{~V} / 0$ & 61 & 5 & 8.2 \\
\hline M694V/Other & 257 & 4 & 1.5 \\
\hline Other/Other & 93 & 1 & 1.1 \\
\hline
\end{tabular}

\section{Discussion}

In this study we report the frequency and type of articular involvement in 687 Armenian pediatric FMF patients. The total frequency was high $56.4 \%$, and we found an especially high number of patients with CA $(4.7 \%)$, who would also qualify for a diagnosis of JIA. Although heterozygous genotype for $M E F V$ mutation was associated with abortive and mild FMF features in general, the carriers of a single M694V mutation more frequently had arthritis, especially CA with features of spondyloarthritis.

Our results suggest that the presence of M694V mutation could be considered a risk factor for articular involvement.
Depending on the type of mutation (homozygous or heterozygous) the patients with CA may present with early, very severe or only atypical manifestations of FMF. The risk of CA was higher in M694V-heterozygotes and M694Vhomozygotes. The frequency of CA was significantly increased also in FMF heterozygotes without M694V mutation compared to compound-heterozygotes. We therefore recommend that JIA patients from an ethnical background with increased FMF-rates should be tested for $M E F V$ mutations, because they may have profit from colchicine therapy for the prevention of amyloidosis.

Homozygosity for the V726A mutation was an important risk factor for articular involvement in our cohort. However, the V726A mutation is relatively rare in the Armenian 
population, and our cohort comprises only 10 patients who were homozygous for this MEFV gene. We therefore think that this result may possibly reflect a bias and it needs to be controlled in a different population.

Our findings are supported by the literature. In earlier study from Israel, which focused on the musculoskeletal manifestations of FMF, arthritis occurred in $71 \%$ of the Sephardic Jewish patients with musculoskeletal symptoms, all of whom carried the M694V mutation, whereas arthritis occurred in only $40 \%$ of the Arab patients, who carried mostly the V726A and M680I mutations [18]. They had shown that not only patients with a classic clinical pattern of the disease bear the M694V mutation, but that this mutation is also found among patients with atypical and severe musculoskeletal manifestations. Therefore, in view of the frequent occurrence of FMF in the population at risk, authors called for the inclusion of genetic screening for FMF in the investigation of children of Mediterranean ancestry who display unexplained recurrent musculoskeletal manifestations [19]. There was shown that that homozygosity for the M694V mutation is predominant among patients originating from North Africa, and is associated with a severe course and prognosis of FMF [18].

In a Turkish population survey, $44 \%$ of the FMF patients bearing the M694V mutation experienced recurrent arthritis, compared with only $9 \%$ of the patients bearing the M680I mutation [20]. As reported, in $2-5 \%$ of FMF patients with protracted arthritis, irreversible changes may occur and in some joint replacement may be indicated. Sacroiliitis may be detected. HLA B27 is positive in less than $40 \%$ of those who manifest features of spondylarthritis [23-25].

Our data are compatible with the hypothesis of a disorder (unlinked to $M E F V$ ) whose severity may be influenced by the FMF mutation, with $M E F V$ acting as a modifier gene. In the presence of a $M E F V$ mutation, individuals who may also have other susceptibility factors for JIA, are at a higher risk to develop chronic arthritis. In our cohort, this seemed to be especially the case for a type of chronic arthritis with features of spondyloarthritis, although the majority of our patients tested negative for HLA B27 [25, 26].

The results of study from Akar S. et al [21] in Turkey not only confirmed an increased prevalence of spondyloarthritis (SpA) and ankylosing spondylitis (AS) among FMF patients, but also indicated that they were more common among the first degree relatives of FMF patients than in the general population. According to their hypothesis, the higher frequency of M694V among FMF patients with radiologically confirmed sacroiliitis suggests that MEFV gene variations may be the pathogenic link between the two disorders. It may be considered a geographic region-specific risk factor for $\mathrm{SpA} / \mathrm{AS}$ affecting a common inflammatory pathway of IL-1 in the pathogenesis of these diseases. Sarikaya S. et al [22] found that FMF has an earlier onset when compared to other rheumatologic diseases and therefore, in patients with peripheral arthritis and spondylitis, FMF should be considered as differential diagnosis and other signs and symptoms of the disease should be investigated, and an analysis for the MEFV gene might also be helpful in suspicious cases. Moreover recent studies reported the increased innate immune system inflammation seen in FMF may be considered as a susceptibility factor since it predisposes to certain inflammatory conditions and FMF is commonly associated with other inflammatory diseases. Therefore, clinicians should be cautious about comorbid inflammatory diseases in FMF patients, particularly in those with persistent inflammation. Identification of pathogenic pathways linking FMF to these diseases warrants further investigations $[27,28]$.

\section{Conclusion}

We recommend, JIA patients from an ethnical background with increased FMF-rates should be tested for MEFV gene mutations, because chronic arthritis may be the first and only atypical manifestation of FMF and occurred usually in patients with severe M694V mutation and they may profit from colchicine therapy for the prevention of amyloidosis. Patients of Armenian origin with refractory course of arthritis should be asked for isolated febrile attacks, hemorrhagic (Schoenlein-Henoch-like) vasculitis, rare episodes of pleuritis and the family history to rule out FMF.

\section{Conflict of Interest}

No potential conflict of interest was reported by the author (s).

\section{References}

[1] Livneh A, Langevitz P, Zewer D, et al. Criteria for the diagnosis of familial Mediterranean fever. Arthritis and Rheumatism 1997; 40: 1879-1885.

[2] Livneh A, Langevitz P Diagnostic and treatment concerns in familial Mediterranean fever. Bailliere's best practice \& research. Clinical rheumatology 2000; 14: 477-498.

[3] Lidar M, Livneh A Familial Mediterranean fever: clinical, molecular and management advancements. The Netherlands journal of medicine 2007; 65: 318-324.

[4] Padeh S, BerkunY Auto-inflammatory fever syndromes Rheum Dis Clin North Am. 2007 Aug; 33 (3): 585- 623..

[5] Stojanov S, Kastner D. Familial autoinflammatory diseases: genetics, pathogenesis and treatment. Current opinion in rheumatology 2005; 17: 586-599.

[6] Rigante D, La Torraca I, Ansuni V, et al. The multi-face expression of familial Mediterranean fever in the child. European review for medical and pharmacological sciences 2006; 10: 163-171.

[7] Mor A, Rivka G, Livneh A Abdominal and digestive system associations of familial Mediterranean fever. The American Journal of gastroenterology 2003; 98: 2594-2604.

[8] Torossyan Y, Aksentijevich I, Sarkisian T, et al. Role of complex allels and gender in the susceptibility to FMF in the Armenian population. Am J Human Genetics 2000; 64: 1275. 
[9] Hayrapetyan A Genetical aspects of familial Mediterranean fever in Armenians. Dissertation, Yerevan State Medical University, 2002.

[10] Sarkisian T, Ajrapetyan H, Beglaryan A, et al Molecular Diagnosis of familial mediterrenean fever in Armenians. The New Armenian Medical Journal 2007; 1: 33-40.

[11] Avagyan T, Amaryan G, Hayrapetyan H, Budumyan A, Tadevosyan A Population based study of frequency of carrying FMF mutation among Armenian females; Pediatric Rheumatology, 2017, 15 (Suppl 2): 64, DOI 10.1186/s12969017-0185-x.

[12] Rabinovich E, Shinar Y, Leiba M, Ehrenfeld M, Langevitz P, Livneh A. Common FMF alleles may predispose to development of Behcet's disease with increased risk for venous thrombosis. Scandinavian journal of rheumatology 2007; 36: 48-52.

[13] Katsicas M, Russo R. Use of infliximab in patients with systemic juvenile idiopathic arthritis refractory to etanercept. Clinical and experimental rheumatology 2005; 23: 545-548.

[14] Lidar M, Kedem R, Mor A, Levartovsky D, et al. Arthritis as the sole episodic manifestation of familial Mediterranean fever. J Rheumatol. 2005; 32: 859-862.

[15] Dusunsel R, Dursum I, Gunduz Z, Poyrazoglu H, et al. Genotype-phenotype correlation in children with familial Mediterranean fever in a Turkish population. Pediatrics international: official journal of the Japan Pediatric Society 2008; 50: 208-212.

[16] Shohat M, Magal N, Shohat T, Chen X, et al. Phenotypegenotype correlation in familial Mediterranean fever: evidence for an association between Met694Val and amyloidosis. European journal of human genetics. 1999; 7: 287-292.

[17] Petty R, Southwood T, Manners P, Baum J, Glass D. et al. International League of Associations for Rheumatology classification of juvenile idiopathic arthritis: second revision, Edmonton, J Rheumatol 2004; 31: 390-392.

[18] Brik R, Shinawi M, Kasinetz L, Gershoni-Baruch R The musculoskeletal manifestations of familial Mediterranean fever in children genetically diagnosed with the disease. Arthritis and rheumatism 2001; 44: 1416-1419.

[19] Brik R, Shinawi M, Kepten I, Berant M, Gershoni-Baruch R.
Familial Mediterranean fever: clinical and genetic characterization in a mixed pediatric population of Jewish and Arab patients. Pediatrics, 1999; 103: e70.

[20] Yalçinkaya F, Cakar N, Misirlioğlu M, Tümer N, Akar N, et al Genotype-phenotype correlation in a large group of Turkish patients with familial mediterranean fever: evidence for mutation-independent amyloidosis. Rheumatology (Oxford) 2000; 39: 67-72.

[21] Akar S, Soysal O, Balci A, Solmaz D, Gerdan V, Onen F, et al High prevalence of spondyloarthritis and ankylosing spondylitis among familial Mediterranean fever patients and their first-degree relatives: further evidence for the connection. Arthritis research \& therapy 2013; 15: R21.

[22] Sarikaya S, Özdolap S, Marsalis E. et al. Spondylitis and arthritis in FMF. Archives of Rheumatology. 2012, Volume 27, Issue 4, Page: 241-247, DOI: 10.5606/tjr.2012.043

[23] Kasifoglu T, Çalısır C, Cansu DÜ, Korkmaz C. The frequency of sacroiliitis in Familial Mediterranean Fever and the role of HLA-B27 and MEFV mutations in the development of sacroiliitis. Clin Rheumatol 2009; 28: 41-6.

[24] Ozdogan H, Ugurlu S. Familial Mediterranean Fever. Presse Med., Quarterly Medical Review 2019; https://doi.org/10.1016/j. lpm.2018.08.014.

[25] Ozen S, Demir S. Monogenic periodic fever syndromes: treatment options for the pediatric patient. Paediatr Drugs 2017; 19: 303-1. doi: 10.1007/s40272-017-0232-6

[26] Manna R, Rigante D. Familial Mediterranean Fever: Assessing the Overall Clinical Impact and Formulating Treatment Plans; Mediterr J Hematol Infect Dis 2019; 11; e2019027.

[27] Balcı-Peynircioğlu B, Kaya-Akça Ü, Serap Arıcı Z, Avcı E et al. Comorbidities in familial Mediterranean fever: analysis of 2000 genetically confirmed patients. Rheumatology (Oxford), 2020, 59 (6), 1372-1380, doi: 10.1093/rheumatology/kez410.

[28] Atas N., Armagan B., Bodakci E, Satis H, Sari A, et al Familial Mediterranean fever is associated with a wide spectrum of inflammatory disorders: results from a large cohort study. Rheumatol Int. 2020 Jan; 40 (1): 41-48. doi: 10.1007/s00296-019-04412-7. 